\title{
The rise of the afterglow in GRB 050820a
}

\author{
F. Genet, F. Daigne, and R. Mochkovitch
}

\author{
Institut d'Astrophysique de Paris - UMR 7095 CNRS et Université Pierre et Marie Curie, 98 bis boulevard Arago, \\ 75014 Paris, France \\ e-mail: genet@iap.fr
}

Received 1 August 2006 / Accepted 4 April 2007

\begin{abstract}
Context. The early optical afterglow of GRB 050820a recorded by the RAPTOR telescope shows both a contribution from the prompt emission and the initial rise of the afterglow.

Aims. It is therefore well-suited for the study of the dynamical evolution of the GRB ejecta when it first undergoes the decelerating effect of the environment. This is a complex phase where the internal, reverse, and forward shocks can all be present simultaneously. Methods. We have developed a simplified model that can follow these different shocks in an approximate, but self-consistent way. It is applied to the case of GRB 050820a to obtain the prompt and afterglow light curves.

Results. We show that the rise of the afterglow during the course of the prompt emission has some important consequences. The reverse shock propagates back into the ejecta before internal shocks are completed, which affects the shape of the gamma-ray profile. Conclusions. We get the best results when the external medium has a uniform density, but obtaining a simultaneous fit of the prompt and afterglow emission is not easy. We discuss a few possibilities that could help to improve this situation.
\end{abstract}

Key words. gamma rays: bursts - shock waves - radiation mechanisms: non-thermal

\section{Introduction}

Just a few GRBs have been captured in the optical while the gamma-ray prompt emission was still active. During the preSWIFT era, the only known example was GRB 990123. Its optical signal was not correlated to the gamma rays (Akerlof et al. 1999) and instead showed a power-law decline $\left(F \sim t^{-2}\right)$, which was interpreted as a contribution from the reverse shock (Sari \& Piran 1999). In the past two years, thanks to the early and accurate localizations by SWIFT and to the progress in groundbased robotic telescopes, a few other cases have been discovered. While in GRB 050401 (Rykoff et al. 2006) and GRB 051111 (Butler et al. 2006) only two data points were recorded during the gamma-ray emission, the TAROT observatory allowed continuous monitoring of GRB $060111 \mathrm{~b}$ for more than $20 \mathrm{~s}$ during the prompt phase (Klotz et al. 2006). In none of these bursts does the optical emission appear correlated to the gamma rays, and GRB 060111b showed an initial power-law decay of slope $\alpha=-2.38 \pm 0.11$, very similar to the behavior of GRB 990123 . Conversely, the optical and gamma-ray light curves were correlated in GRB 041219a (Vestrand et al. 2005), indicating that, at least in some bursts, the prompt component could also be detected in the visible.

The case of GRB 050820a is especially interesting because, in addition to a prompt component, the visible light curve exhibited a sharp rise at about $250 \mathrm{~s}$, which probably marks the onset of the afterglow. RAPTOR observations started $18 \mathrm{~s}$ after the BAT trigger that was caused by a faint precursor $200 \mathrm{~s}$ before the main pulses in the burst profile. Vestrand et al. (2006) interpret the RAPTOR observations with a phenomenological function describing the rise of the afterglow with an additional component proportional to the prompt KONUS gamma-ray profile. We use here a more detailed description of the burst dynamical evolution to see if it can reproduce the observations both in the gamma and visible ranges.

\section{The burst model}

We have developed an approach that allows us to simultaneously follow the internal, reverse, and forward shocks in GRBs. This is crucial for representing the early afterglow where these three kinds of shocks can coexist and contribute to the observed emission. Internal shocks are treated by using a large number of discrete shells to represent the relativistic flow emerging from the central engine (Daigne \& Mochkovitch 1998a). Fast shells catch up and collide with slower ones, the dissipated energy being shared between baryons, electrons (fraction $\epsilon_{e}$ ), and the magnetic field (fraction $\epsilon_{B}$ ). Electrons then radiate by the synchrotron process at a characteristic energy $E_{\mathrm{syn}}$, which depends on the assumed values of $\epsilon_{e}$ and $\epsilon_{B}$. The spectrum is a broken power law of respective (photon) index $\alpha$ and $\beta$ below and beyond $E_{\text {syn }}$. We have adopted $\beta=-2.25$ (Preece et al. 2000) and considered different possible values for $\alpha$ between $-3 / 2$ and $-2 / 3$ (see Sect. 3.2).

The interaction with the environment is implemented by considering the contact discontinuity that separates the ejecta and the shocked external medium. In our simple description, it is represented by two shells moving at the same Lorentz factor $\Gamma$. The first one corresponds to the part of the ejecta already crossed by the reverse shock (of mass $M_{\mathrm{ej}}$ ) and the second to the shocked external medium (of mass $M_{\mathrm{ex}}$ ). Two processes affect this twoshell structure at the contact discontinuity: it collides either with shells of the external medium at rest or with rapid shells from the relativistic ejecta catching up. This represents both the forward and reverse shocks in our simplified picture (Genet et al. 2006a).

The interaction with the external medium is discretized by assuming that a collision occurs each time the contact 

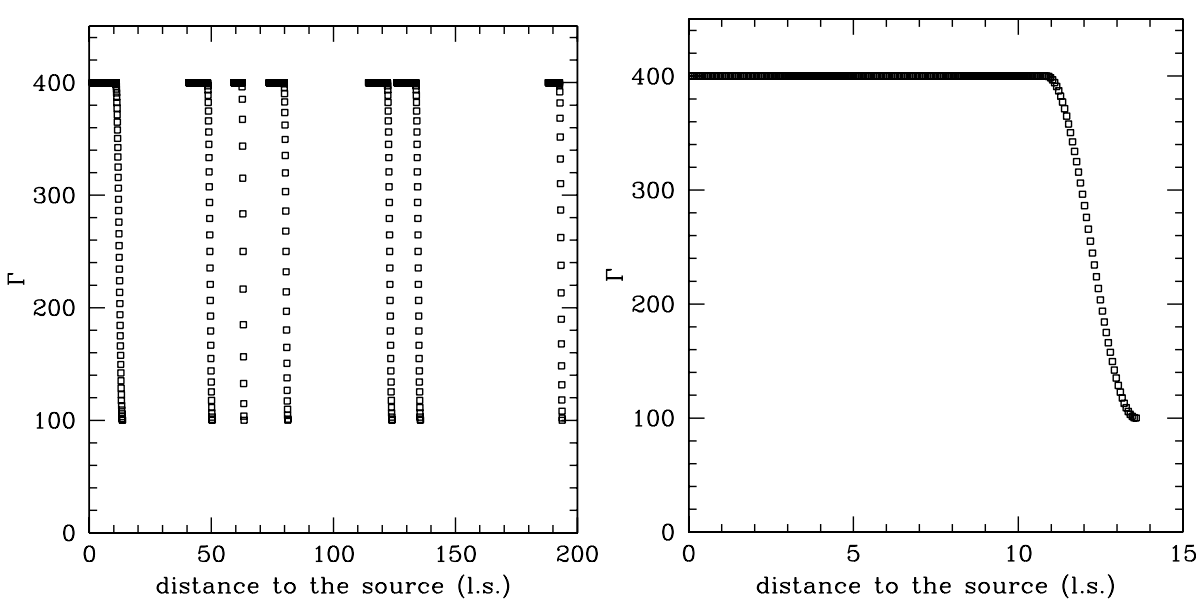

Fig. 1. Left: initial distribution of the Lorentz factor in the relativistic flow. The $\Gamma$ value of each shell is given as a function of the distance to the source in light seconds. Right: zoom on the last episode of wind production showing the transition between the slow and fast material. discontinuity has travelled from a radius $R$ to a radius $R^{\prime}$, so that the swept-up mass is

$m_{\mathrm{ex}}=\int_{R}^{R^{\prime}} 4 \pi r^{2} \rho(r) \mathrm{d} r=q \frac{M}{\Gamma}$

where $M=M_{\mathrm{ej}}+M_{\mathrm{ex}}$, and $\rho(r)$ is the density of the external medium. We have taken $q=10^{-2}$, which represents a good compromise between numerical accuracy and computing time.

\section{The prompt emission of GRB 050820a}

\subsection{Prompt gamma-ray emission}

To compute the prompt emission of GRB 050820a, we start from an initial distribution of the Lorentz factor in the flow ejected by the central source, which can lead to the observed gammaray profile. Such a distribution is shown in Fig. 1. It is made of several episodes of wind production for a total duration of about $200 \mathrm{~s}$ in the source rest frame. In each episode "slow" material $(\Gamma=100)$ is emitted first and then followed by some more rapid one $(\Gamma=400)$ with a transition of the form

$\Gamma(t)=250-150 \cos \left[\pi\left(\frac{t-t_{0}}{t_{\mathrm{m}}-t_{0}}\right)\right]$

where $t_{0}$ is the starting time of the episode and $t_{\mathrm{m}}$ the time when $\Gamma(t)$ reaches its maximum value of 400 . Such a cosine form has been used in our previous works (Daigne \& Mochkovitch 1998b, 2000) and provides a smooth transition between the rapid and slower parts of the flow. The kinetic energy injected in the different episodes is fixed to reproduce the intensity of the successive spikes in the gamma-ray profile. To account for the high isotropic gamma-ray energy of GRB 050820a $\left(E_{\gamma}^{\text {iso }} \sim\right.$ $8 \times 10^{53} \mathrm{erg}$, Golenetskii et al. 2005), we had to inject an even higher kinetic energy $E_{\mathrm{K}}^{\text {iso }}=1.8 \times 10^{55}$ erg into the flow since the global efficiency $f$ of the conversion process is low. We have $f=f_{\text {diss }} \times \epsilon_{e} \lesssim 5 \%$, where $f_{\text {diss }} \lesssim 15 \%$ is the efficiency for dissipation by internal shocks. We have assumed a high $\epsilon_{e}=0.33$ to still have a reasonable total efficiency, since only the fraction of the energy transferred to electrons is finally radiated. We compare the resulting synthetic profile (obtained with a low energy index $\alpha=-1$ ) to the KONUS light curve in Fig. 2, neglecting at this stage the effect of the external medium. The agreement is satisfactory, as the main objective of this work is not to reproduce the temporal behavior of GRB 050820a accurately but to study the rise of the afterglow in a complex burst.

\subsection{Prompt optical emission}

The RAPTOR observations show a contribution from the prompt emission in the optical that becomes blended with the afterglow after about $200 \mathrm{~s}$. The prompt emission of GRBs must take place in the fast cooling regime to guarantee that the energy dissipated by internal shocks is efficiently radiated. The expected emission spectrum should then have a spectral index of $\alpha=-3 / 2$ for $v_{\mathrm{c}}<v<v_{\mathrm{m}}$ and $\alpha=-2 / 3$ for $v<v_{\mathrm{c}}$, where $v_{\mathrm{m}}$ is the characteristic synchrotron frequency and $v_{\mathrm{c}}$ the cooling frequency that typically lies in the optical/UV range (Sari et al. 1998). However such a spectrum contradicts the majority of observed GRB spectra (Ghisellini et al. 2000) where the typical low-energy photon spectral index $\alpha$ is closer to -1 than to -1.5 (Preece et al. 2000). A more detailed description of the emission processes would therefore be necessary to obtain the prompt optical flux in a fully reliable way. Here we have simply adopted a single, averaged low-energy spectral index $\langle\alpha\rangle$ from the gamma to the optical energy ranges. The resulting prompt optical flux is then very sensitive to the value of $\langle\alpha\rangle$ as shown in the left panel of Fig. 3 where the $R$ magnitude light curve has been represented for $\langle\alpha\rangle=-2 / 3,-1$ and $-3 / 2$. When $\langle\alpha\rangle$ increases from $-3 / 2$ to $-2 / 3$, the R flux decreases by about $10 \mathrm{mag}$. Since the prompt contribution to the RAPTOR light curve peaks at $R \sim 15$, it indicates a value of $\langle\alpha\rangle \sim-1.15$. We also find that the profile shape evolves, becoming less spiky at larger $\langle\alpha\rangle$. This can be understood since the flux in the visible is proportional to $\left(\frac{E_{R}}{E_{\mathrm{syn}}}\right)^{\langle\alpha\rangle+1}$, where $E_{R} \sim 1 \mathrm{eV}$ is a typical energy for the $\mathrm{R}$ band. Comparing the fluxes for two different values of $\langle\alpha\rangle$ yields

$$
\frac{F_{R}^{\left\langle\alpha_{1}\right\rangle}}{F_{R}^{\left\langle\alpha_{2}\right\rangle}}=\left(\frac{E_{R}}{E_{\mathrm{syn}}}\right)^{\left\langle\alpha_{1}\right\rangle-\left\langle\alpha_{2}\right\rangle}
$$

If $E_{\text {syn }}$ stayed constant during burst evolution, the light curves for different $\langle\alpha\rangle$ would be simply proportional. However, since $E_{\text {syn }}$ is correlated to intensity, the flux ratio varies: for $\left\langle\alpha_{1}\right\rangle\left\langle\left\langle\alpha_{2}\right\rangle\right.$, it increases at intensity peaks.

\section{Afterglow calculation}

We first obtained afterglow light curves for a uniform external medium. The general shape of the afterglow consists of an initial weak bump produced when the material responsible for the gamma-ray precursor hits the ISM. It is followed by a sharp rise leading to the second bright bump when the material making the main peaks of the profile catches up with the forward shock. The right panel of Fig. 3 shows the evolution of the afterglow when 

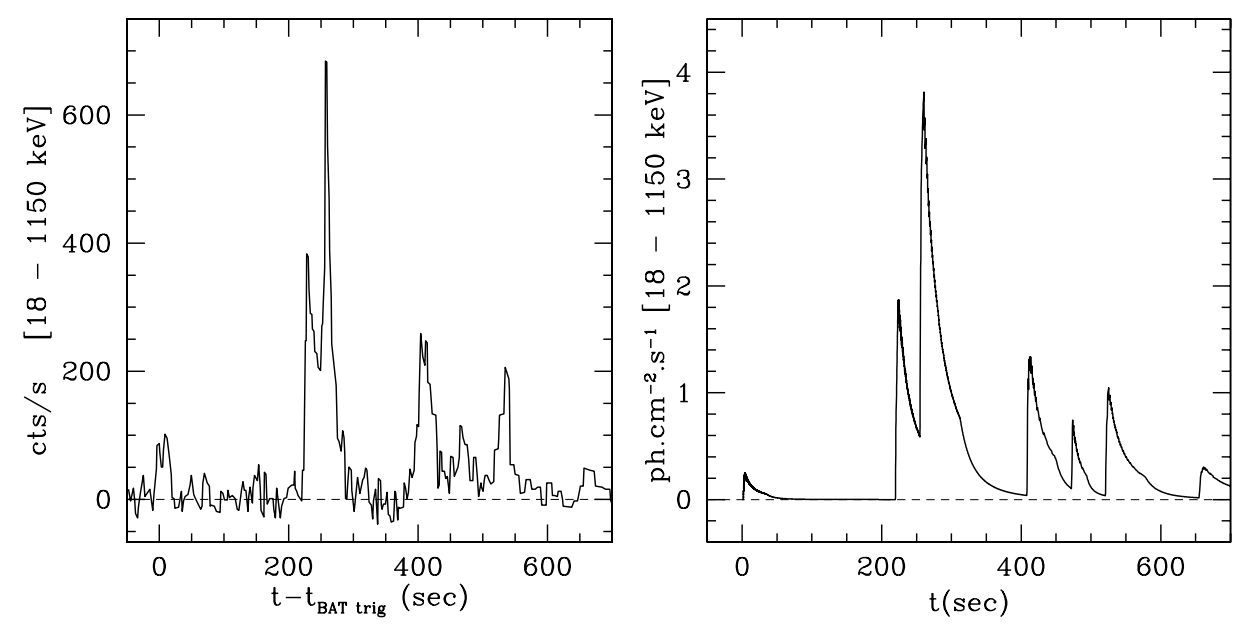

Fig. 2. Comparison of the KONUS light curve (left) to our synthetic profile (right) in the same energy band: $18-1150 \mathrm{keV}$.
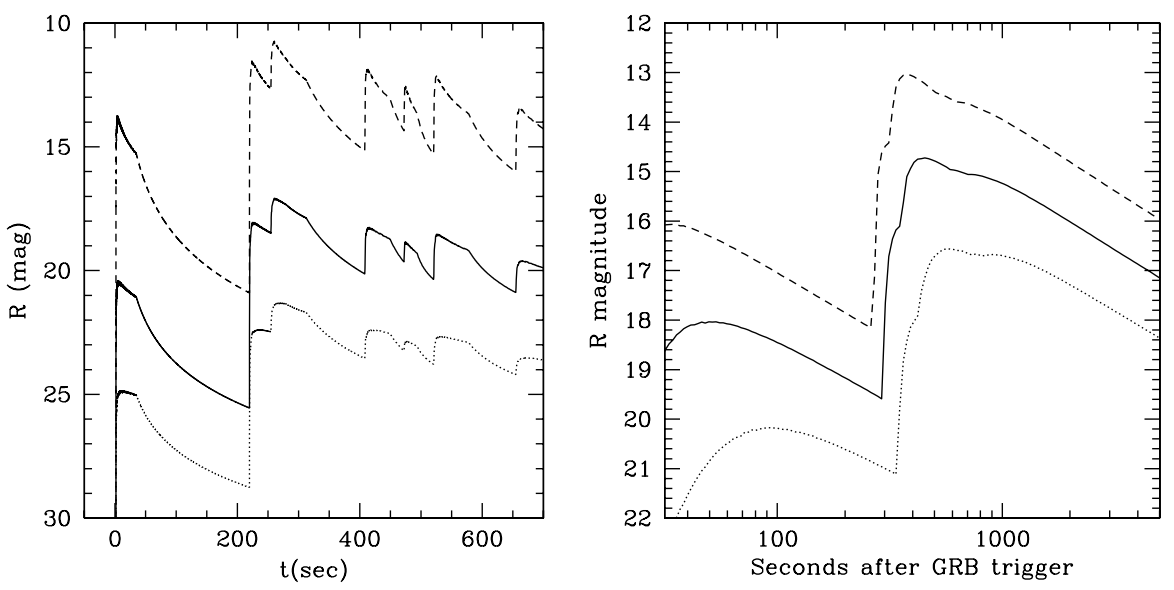

Fig. 3. Left: prompt light curves in the $R$ band for $\langle\alpha\rangle=-2 / 3$ (dotted line), -1 (full line), and -1.5 (dashed line); right: afterglow light curves for $\epsilon_{e}=3 \times 10^{-3}, \epsilon_{B}=10^{-5}, p=2.5$, and different densities in the external medium, respectively $n=0.3$ (dotted line), 3 (full line), and $30 \mathrm{~cm}^{-3}$ (dashed line).

the external density $n$ is varied. Increasing $n$ naturally leads to a brighter afterglow with an earlier rise of the second bump. Once the density has been fixed by the time of the onset of the second bump, the microscopic parameters $\epsilon_{e}, \epsilon_{B}$, and $p$ can be adjusted to get the correct intensity and decay slope after maximum. A good compromise appears to be $n=3 \mathrm{~cm}^{-3}, \epsilon_{e}=3 \times 10^{-3}$, $\epsilon_{B}=10^{-5}$, and $p=2.5$.

We then considered a stellar wind environment, which should be expected if long bursts are produced by exploding WR stars. An example of the resulting afterglow is given in Fig. 4. It can be seen that the first bump is now much too bright since the first part of the ejecta encounters the densest part of the wind, close to the star. The RAPTOR observations (where the first bump is 3 mag fainter than the second) then clearly favor a uniform environment except if some special circumstances (varying microphysics parameters, pair loading and pre-acceleration of the external medium) can strongly reduce the first bump's contribution.

\section{Effect of the environment on the burst prompt emission}

The afterglow light curve obtained with $n=3 \mathrm{~cm}^{-3}$ fits the RAPTOR data reasonably well, as shown in Fig. 5 where we also represent the prompt emission component for $\langle\alpha\rangle \sim-1.15$. However the rise of the afterglow at $t \sim 250 \mathrm{~s}$, i.e. during the prompt phase, has some important consequences. The reverse shock resulting from the early deceleration of the ejecta affects

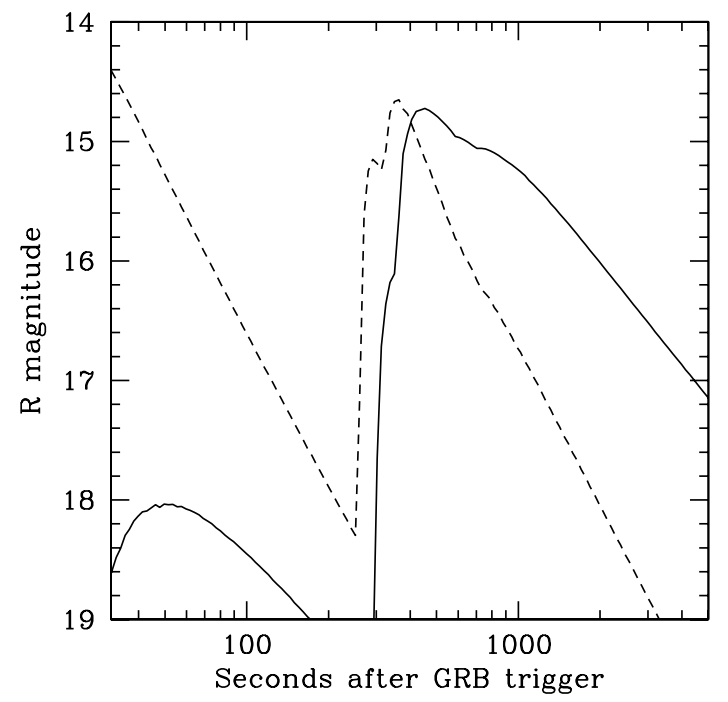

Fig. 4. Comparison of the afterglow light curve for a wind with $A_{*}=0.1$ (dashed line) and a uniform medium with $n=3 \mathrm{~cm}^{-3}$ (full line).

the distribution of the Lorentz factor well before internal shocks are completed. This is turn modifies the gamma-ray profile as shown in Fig. 6, where the profile with $n=3 \mathrm{~cm}^{-3}$ is compared to the $n \rightarrow 0$ case already shown in Fig. 2. After $300 \mathrm{~s}$, new pulses are present, the photon flux is larger, and the similarity with GRB 050820a is partially lost. Reducing the density of the 


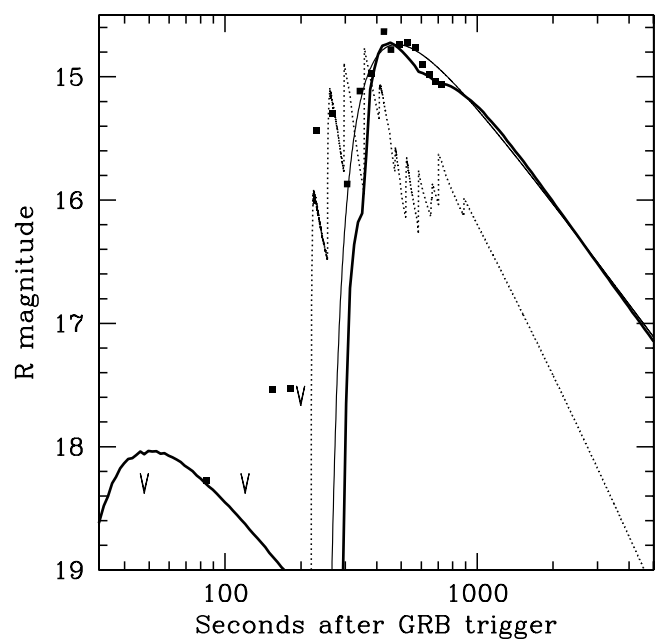

Fig. 5. Results of our model compared to the RAPTOR observations. The thick full line is our synthetic afterglow light curve (external shock contribution) and the dotted line is the prompt optical component calculated with $\langle\alpha\rangle \sim-1.15$ (internal shock contribution). RAPTOR data (Vestrand et al. 2006) is shown as squares or V signs (upper limits). The thin full line is a phenomenological function used by Vestrand et al. to represent the afterglow component.

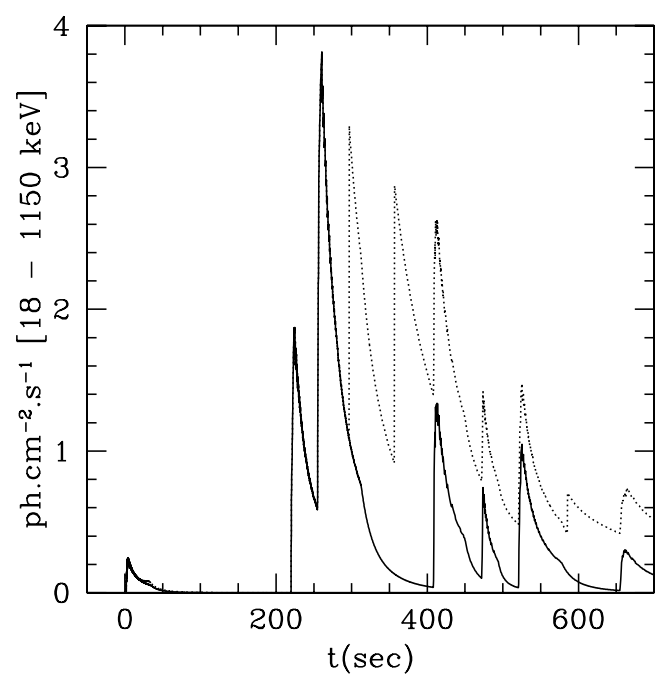

Fig. 6. Predicted gamma-ray profile when the effect of the external medium is included (for $n=3 \mathrm{~cm}^{-3}$, dotted line) compared to the original profile with no external medium (full line).

external medium tends to better preserve the burst profile but also shifts the rise of the afterglow to later times (see Fig. 3).

\section{Discussion}

The simplified model we have developed neglects many important aspects of the hydrodynamical evolution of the relativistic outflow produced by the burst central engine (e.g. pressure waves, radial structure of flow). However, within these approximations, it allows us to study the complete evolution with one single, self-consistent calculation including both the phase of internal shocks and the deceleration by the external medium. It is therefore ideally suited for GRB 050820a where the afterglow starts to rise before the end of internal shocks.

We have shown that obtaining a simultaneous fit for both the prompt and afterglow emission of GRB 050820a is not easy because, due to the early deceleration of the flow, the reverse shock gets mixed with internal shocks, which affects the burst profile in a complicated way. We are therefore currently examining a few possibilities that could improve the situation:

(i) The most obvious solution would be to start with an initial distribution of the Lorentz factor different from the one shown in Fig. 1 so that, after it had been perturbed by the reverse shock, it would finally lead to the observed gammaray profile. Preliminary calculations indicate that it should be possible to recover the main spikes in the profile, but also difficult to avoid a few additional weak pulses partially overlapping with them.

(ii) Another possibility could be to include in the calculation the pair-loading process resulting from the gamma-ray flash that preaccelerates the circumstellar medium (Madau \& Thomson 2000; Beloborodov 2002). The low-density cavity that forms around the source will reduce the effect of the reverse shock. It will however also delay the rise of the afterglow by an amount that should remain compatible with the observations.

(iii) One could finally consider the electromagnetic model (EMM) proposed by Lyutikov \& Blandford (2003) rather than the standard model. The EMM has no reverse shock, but its physics is also more complex and uncertain so that the comparison with observations is not straightforward. Using a simple model to compute GRB afterglows in the context of the EMM (Genet et al. 2006b), we have found that good fits of the GRB 050820a afterglow can be obtained if electromagnetic energy is released in two steps: a weak precursor followed by the main event. However, we are unable to calculate the related prompt optical and gamma-ray emissions that, in the EMM, result from magnetic reconnection processes. It is therefore impossible to check the overall consistency of the model.

\section{Conclusion}

We have developed an approximate method that can follow the complex dynamical evolution of GRB ejecta during the early afterglow phase when the internal, reverse, and forward shocks can be simultaneously active and contribute to the observed radiation.

It has been applied to GRB 050820a, for which RAPTOR observations show both a prompt emission component and the onset of the afterglow. We have found that the rise of the afterglow during the prompt phase implies that the reverse shock is active well before internal shocks are completed. This makes a simultaneous fit of the prompt gamma-ray emission and optical afterglow rather challenging, the best results being however obtained for a uniform external medium. We have briefly discussed possible ways to improve the situation in the context of the standard internal/external shock model but also considered the EMM as an alternative.

As more bursts are captured in the optical during the prompt phase and very early afterglow, it should become possible to study in more detail this complex period where the burst energy starts to be transferred to the surrounding medium. In X-rays it is often characterized by a shallow decline that also challenges simple models. Multiwavelength observations showing the rise of the afterglow will then be of prime interest to see if it can still be explained by the standard internal/external shock model or if changes in the current paradigm will be necessary. 


\section{References}

Akerlof, C. W., Balsano, R., Barthelmy, S., et al. 1999, Nature, 398, 400 Beloborodov, A. M. 2002, ApJ 565, 808

Butler, N. R., Li, W., Perley, D., et al. 2006, ApJ, 652, 1390

Daigne, F., \& Mochkovitch, R. 1998a, A\&A, 358, 1157

Daigne, F., \& Mochkovitch, R. 1998b, MNRAS 296, 275

Genet, F., Daigne, F., \& Mochkovitch, R. 2006a, in Gamma-Ray Bursts in the Swift Era, AIP Conf. Proc., 838, 313

Genet, F., Daigne, F., \& Mochkovitch, R. 2006b, A\&A, 457, 737

Ghisellini, G., Celotti, A., \& Lazzati, D. 2000, MNRAS, 313, L1
Golenetskii, S., et al. 2005, GCN No. 3852

Klotz, A., et al. 2006, A\&A, 451, L39

Lyutikov, M., \& Blandford, R. D. 2003, [astro-ph/0312347]

Madau, P., \& Thomson, C. 2000, ApJ, 534, 239

Preece, R. D., et al. 2000, ApJS, 126, 19

Rykoff, E. S., et al. 2005, ApJ, 631, L121

Sari, R., \& Piran, T. 1999, ApJ, 520, 641

Sari, R., Piran, T., \& Narayan 1998, ApJ, 497, L17

Vestrand, J. A., et al. 2005, Nature, 435, 178

Vestrand, J. A., et al. 2006, Nature, 442, 172 